\title{
Research on Optimizing Design for Diffuser-Tower Structure of Primary Fan in Shaft
}

\author{
S. Q. Chen *, H. Q. Wang, Y. C. Li \\ School of Energy \& Safety Engineering, Hunan University of Science and Technology, Xiangtan 411201, \\ China \\ Email: zunyichsq@163.com
}

\begin{abstract}
Diffuser-Tower (DT) is an equipment reclaiming energy of primary fan (PF) in shaft. The structure of DT is closely correlative to energy degradation of $\mathrm{PF}$, however reasonable inner flow field is related to DT structure. Energy-waste of PF occupies 1/3 production energy-waste in mining industry, and ventilation of electric power wasted occupies $70 \%$ energy-wasted PF [1]. Main are appraisal parameters of DT's performance are section enlarge ratio (SER), recovery ratio of static pressure (RSP), DT's diffusing efficiency (DDE) of PF, Local resistance ratio (LRR) evenness degree of velocity distribution in DT's outlet. The outlines of new type streamline DT are based on repeated addition to non-uniform paralleled plane airflow with sink flow with considering sunk practice of airflow after PF [2]. In the condition of same velocity in inlet and
\end{abstract}

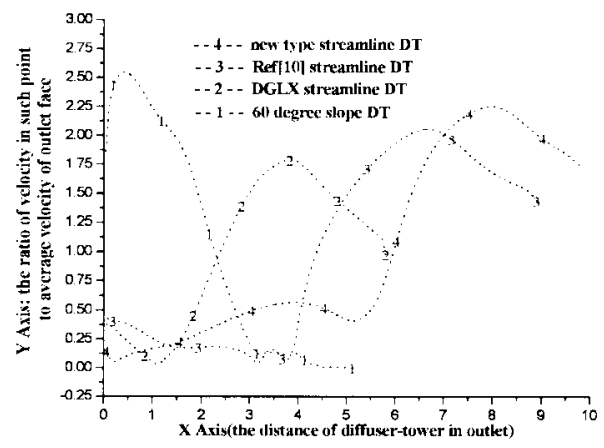

Figure 1: The Graph of Distributing on Velocity in Outlet

Table 1: Main Performances of Four Type Diffuser-Tower

\begin{tabular}{cllll}
\hline TYPE & SER & RSP & LRR & DDE \\
\hline $60^{\circ}$ Slope & 1.290 & 0.0142 & 0.5696 & 0.0243 \\
DGLX Streamline & 2.146 & 0.1044 & 0.6773 & 0.1336 \\
Ref[3] Steamline & 2.691 & 0.4162 & 0.4452 & 0.4832 \\
New type Strealine & 2.963 & 0.4466 & 0.4389 & 0.5044 \\
\hline
\end{tabular}

flow rate, there are in turn simulated the flow fields of $60^{\circ}$ slope DT, DGLX DT, Ref [3] streamline DT and new type streamline DT. See Table 1 results of In the condition of same needed flow rate on shaft ventilation system, new type streamline DT is easier to achieve saving-energy and degradingconsumption performance of PF because of owning larger section enlarge ratio to its self. DT's evenness degree of velocity distribution in outlet is also important appraisal index of DT's performance. See Figure 1 velocity distributions in four type DTs in outlet. It is necessary that the balance point should be probed into between addition of resistance and benefit of homogenization from flow deflectors in way of differentiation. Diffusing efficiency of DT is synthetically embodies from SER, LRR and recovery RSP. Diffusing efficiency is higher as it is easier to achieve saving-energy and degradingconsumption performance of PF. Diffusing efficiency is improved favorably compared designed new type streamline DT, which has such perspective of engineering practice, with other types DT. To some extent, the paper may guide and make reference to the design of DT.

\section{REFERENCES}

1. Zhao ZC, Xie XP. A review on development of theory and technology of mine ventilation. Yunnan Metallurgy, 2002;31(3):23-37

2. Tian RX, Ge YB, Wu WQ. Multigrid method for solving two dimensional incompressible N-S equation using stream function formulation. Journal of Engineering Thermophysics, 2005; 10(4):31-34

3. Tao F. Designing research for reasonable flow pattern of curved diffuser. Energy Technology, $2002 ; 23(4): 147-150$ 